\title{
Physical Aspects of the EEG in Schizophrenics
}

\author{
Thomas Elbert, Werner Lutzenberger, Brigitte Rockstroh, \\ Patrick Berg, and Rudolf Cohen
}

\begin{abstract}
Physical and dynamic aspects of the electroencephalogram (EEG) were evaluated in 12 schizophrenic patients and 12 matched healthy control subjects by means of two descriptive measures proposed by Hjorth (complexity and mobility) and by a nonlinear measure, dimensional complexity. These measures were compared to power spectra analyses. EEG was recorded from frontal, central, and parietal leads under resting conditions (eyes open and eyes closed) for 12 epochs each of $25 \mathrm{sec}$. Patients showed the expected increased activity in the 1-7 Hz band and, furthermore, a scalp distribution of dimensional complexity and Hjorth complexity opposite to the distribution in controls: in patients dimensional complexity yielded higher values at frontal $(F z)$ than central $(C z)$ leads, whereas the resemblance to sinusoidal waveshape (Hjorth complexity) was larger at $F z$ than $C z$. Results indicate more dynamic complexity or variables determining the dynamics of brain processes in frontal areas in patients.
\end{abstract}

\section{Introduction}

Prima vista electroencephalogram (EEG) traces from schizophrenics cannot be discriminated from those of healthy controls. Nevertheless there has been a continuous effort to discover and specify electrocortical substrates for cognitive, emotional, and behavioral abnormalities in schizophrenic patients on the basis of spontaneous EEG records as well as of evoked potentials. As EEG aspects can indicate cognitive and behavioral processes, it seemed straightforward to search for psychopathological indicators. Common findings in schizophrenic patients (as compared to controls) were increased occurrence of abnormal EEG patterns, less alpha and more slow activities, shifts in the center frequency in the alpha band to lower, in the beta band to higher center frequency, less amplitude but more frequency variability, etc. (for summaries see Itil 1997; Shagass 1976; Shagass et al 1982, 1984; Zahn 1986). When hemispheric specificities were analyzed more closely, the balance between left and right hemispheres in the power and céntroid of various EEG bands were reported to differ between schizophrenic patients and controls (see Flor-Henry 1988; Koukkou and Manske 1986; Koukkou et al 1991; Michelogiannis et al 1991). Finally, computerized analyses of multichannel recordings have become available and differences in the scalp topography between schizophrenics and controls have been subsequently reported for spontaneous (e.g., John 1973) as well as evoked activity (Maurer

From the Institute for Experimental Audiology, University of Münster (TE); the Institute of Psychology, University of Tübingen (WL); and the Institute of Psychology, University of Konstanz (BR, PB, RC), Germany.

Address reprints to Prof. Dr. Brigitte Rockstroh, Fachgruppe Psychologie, Postfach 5560, D 7750 Konstanz, Germany.

Received November 24, 1991; revised June 11, 1992. 
and Diercks 1987). Although new information seemed to be added with every new method and although some specification of EEG patterns for subgroups of patients or relationships between those quantitative EEG measures and diagnostic criteria were reported (e.g., Shagass et al 1982, 1984), it was still repeatedly mentioned that results, though reliable, were not specific enough to specifically elucidate schizophrenic psychopathology, or that differences were only statistical in nature, meaning that even the most deviant behavior observed in schizophrenics may, at times, also be observed in an absolutely unremarkable subject. As pointed out by Hjorth (1986), traditional EEG parameters as derived, for example, from power spectrum analysis, do not allow the description of basic rules that characterize the dynamics of the observed EEG potential, that is, the potential changes over time or the set of rules governing the system's state at a given time. More recently, progress in nonlinear-systems theory has introduced new technologies into computerized EEG analyses (reviewed by Pritchard and Duke in press a,b). Over the last decade a new way of understanding the growth of complexity in nature has arisen from physics and biology. Where formerly an erratically behaving variable could be described as random or stochastic, we can now detect patterns and laws, still complex, but much simpler than previously considered. The solution to the enigma created by the linear approximation of the world is nonlinear dynamics, often referred to as chaos or deterministic chaos.

The present paper describes an approach to evaluate measures of the dynamic features of the EEG and compare them with conventional results of EEG power spectra analyses. The nonlinear measure of dimensional complexity should provide insight into EEG dynamics by indicating the number of variables that determine the system's state. In addition, two measures proposed by Hjorth (1986) to describe physical aspects of the EEG trace, complexity, that is, sinusoidality or regularity of the EEG waveshape, and mobility corresponding to the center frequency, were examined. Because these measures have not been applied very often in psychiatric research, it seems necessary to briefly summarize these measures in the following paragraph. It seemed worthwhile to consider nonlinear measures of the EEG such as the dimensional complexity in schizophrenic patients, because such measures might serve as indicators of specific brain functioning in schizophrenia and might in particular elucidate dynamic characteristics of the schizophrenic brain. The EEG was recorded under resting conditions, because it may be assumed that different brain regions are governed by the same dynamic processes if they are not activated by particular tasks and, hence, individual patterns might become prominent.

\section{Methodological Background}

Hjorth's (1986) measures are related to the physical appearance of the EEG waveform: complexity is defined as the average "sharpness" of the tracing that can be regarded as a measure of frequency spread of sinusoidal waves; a pure sine wave yields zero complexity. Mobility expresses the mean (rms) slope of the EEG as mean (rms) amplitude per unit of time and is equivalent to the mean (rms) frequency of the power spectrum.

The algorithms provided by nonlinear systems theory to transform dynamic features into a static picture can be viewed as another mathematical transformation. One of the concepts to describe a dynamic system is that of an idealized space, the state space. A point in the state space characterizes the system at any given moment in time. The exact location of a swinging pendulum at one instant, for example, does not completely characterize its current state. We also need to know its past, for instance, its velocity. A 
point in the state space consisting of two dimensions "position" and "velocity," however, completely describes the current state. By tracing the trajectory of a given system in its state space, the characteristics of a dynamic system are transformed into a static picture. If the system is left unperturbed, its trajectories will be attracted to a subset of points, which is commonly referred to as an attractor. One measure that characterizes an attractor is its dimension. (For example, a pendulum will eventually stop swinging due to friction. In state space it will spiral towards the origin, where the velocity will be zero and the location constant, leading to a point attractor that has zero dimension.) The methods implemented to estimate the dimension (such as the pointwise) do not give the dimension with absolute accuracy. Therefore, it has been referred to as dimensional complexity as structures with higher dimensions are generally viewed as being more complex (see Pritchard and Duke in press a,b). Thus, dimensional complexity refers to the description of the geometrical structure of an attractor or the number of independent entities that determine the system's state at any given point in time. The dimension of the state space corresponds to the number of differential equations of the system, whereas the dimensionality of the attractor is defined as the section of the state space that is occupied by the trajectories or data points after the transitory phase (for tutorial reviews see Pritchard and Duke in press a,b).

Dimensional analysis has been applied to the EEG beginning in the mid-1980s. Lower dimensions have been found in the relaxed state, particularly when the eyes were closed (e.g., Dvorak et al 1986, Graf and Elbert 1989; Layne et al 1986; Rapp et al 1985, 1989), whereas dimensional complexity increases when subjects are given a mental task to perform (Dvorak et al 1980; Nan and Jinghua 1988; Rapp et al 1989; Ray et al 1991; Lutzenberger et al 1992). Other findings include a lowering of dimension during epileptic seizures (Pijn et al 1991), stage 2 sleep, stage 4 sleep and anesthesia, but not rapid eye movement (REM) sleep relative to waking EEG (Babloyantz et al 1985; Watt and Hameroff 1987). Comparing the results from dimensionality analysis with those from traditional Fourier analyses on a variety of mental tasks, it could be shown that conventional measures based on power spectra extract only limited information from the EEG (Lutzenberger et al 1992).

\section{Methods}

\section{Subjects}

EEG recordings were obtained from 12 patients with a schizophrenic disorder according to DSM-III-R (eight men, four women, mean age $26.8 \pm 1.3 \mathrm{yrs}$ ) and 12 control subjects ( 7 men, 5 women, mean age $28.4 \pm 1.5 \mathrm{yrs}$ ). ${ }^{1}$ Inpatients were under neuroleptic medication with a mean of $385.5 \pm 61.4 \mathrm{mg} \mathrm{CPZ}$-equivalent (range 130-813 mg). Two scores describing negative symptoms, "affective flattening" and "cognitive impairment," were based on a principal components analysis (PCA) of all ratings from the Brief Psychiatric Rating Scale (BPRS) (Lukoff et al 1986), the Scale for the Assessment of Negative Symptoms (SANS) (Andreasen 1981), the Positive and Negative Syndrome Scale (PANSS) (Kay et al 1987), and the Psychological Impairment Rating Schedule 
(PIRS) related to negative symptoms of 102 schizophrenic patients from which our subjects were a subsample. It was assured that control subjects were not under psychopharmacological medication.

\section{Physiological Recordings}

The EEG was recorded while subjects were sitting quietly and relaxed in a reclining chair without any particular task. During half of the recording epochs subjects were asked to keep their eyes open but to avoid blinks and eye movements, the other half of the recording epochs were registered with eyes closed. The entire recording lasted for about 4-5 min.

The EEG was recorded from frontal $(\mathrm{Fz})$, central $(\mathrm{Cz})$, and parietal $(\mathrm{Pz})$, leads referenced to linked mastoids. Chlorided Grass silver-cup electrodes were affixed with Grass EC2 electrode cream as a conducting agent. The vertical electrooculogram (EOG) was monitored for ocular artifacts. Beckman silver-silverchloride electrodes were affixed about $1 \mathrm{~cm}$ above and below the left eye. Grass EC2 electrode cream served as the conducting agent. A single ground electrode was attached to the forehead. The skin below each electrode was prepared by cleansing with alcohol. Twelve epochs of 25 -sec each, six with eyes open and six with eyes closed, were recorded.

\section{Apparatus}

EEG signals were acquired using an HP 1000 computer; a Van Gogh 50,000 electroencephalograph was used for the amplification, analog filtering, and display of EEG and EOG data. All channels were amplified with a bandwidth from $0.016 \mathrm{~Hz}$ to $15 \mathrm{~Hz}$ and sampled at a rate of 100 per sec. The high pass frequency cutoff was subsequently raised by digital filtering to $0.5 \mathrm{~Hz}$. Data was transferred to a DEC VAX-station 2000 for subsequent analysis.

\section{Data Reduction and Analysis}

For every subject four EEG epochs of each condition (eyes open, eyes closed) free of large saccadic eye movements were selected. The contamination through eye blinks and small saccades was reduced by subtracting a constant fraction of the EOG from each of the EEG channels. The transmission coefficients were determined as regressions for each of the EEG channels on the EOG channel during epochs when eye movements were present. The median of these epochs was chosen as the coefficient. Such a procedure generally leaves less than $3 \mu \mathrm{V}$ of blink-related activity remaining in the EEG channels (Berg 1986). The length of each of the EEG-traces was 2500 points.

The following measures were calculated for every EEG trace for each of the 24 subjects:

1. A measure of $E E G$ alpha was obtained as the average log power in the range from $8-12 \mathrm{~Hz}$. The power spectrum was calculated by averaging the Fourier transforms of 15 overlapping 2-sec segments (256 points), using Parzen windows on the 2-sec segments.

2. As a measure for the activity in the slow frequency band, the average log power from $1-7 \mathrm{~Hz}$ was determined.

3. Mobility, according to Hjorth, expresses the mean slope of the EEG tracing and corresponds to the mean frequency of the EEG power spectrum. The time unit is calibrated such that the mobility will express the frequency of a pure sine wave in $\mathrm{Hz}$; when the 
tracing is not a pure sine wave it expresses the mean (rms) frequency of the power spectrum.

4. EEG Hjorth complexity is described by Hjorth (1975) as a measure of frequency spread or average "sharpness" of the tracing; a pure sine wave, for instance, yields zero complexity. Thus, more deviancy from a stationary sine wave will produce higher values of the Hjorth complexity measure. (For the mathematical implementation see Hjorth 1975, 1986).

5. Dimensional complexity of the EEG describes the fractal dimension of the presumed attractor of the EEG-generating system. Assuming such an attractor, it can be reconstructed from the EEG time series (in the present study, for instance, 2500 data points per single trace). The first step for this reconstruction is a singular-value decomposition based on the autocovariance function (or autocorrelation function that, based on the subsequent normalization, yields the same results).

Analogous to the way a Fourier transformation can separate a signal into components with common properties, a singular value decomposition (or principal component analysis, PCA) can be used to separate certain properties of the time series into orthogonal components. These properties are selected, for instance, in the usual PCA with event-related potentials by generating a covariance or correlation matrix across conditions or recordings. In the present study we were interested in the time-varying properties of the EEG trace. Therefore, the matrix was generated as an autocovariation function that was created by computing the covariance of the EEG trace with time-shifted copies of the same signal (step 1 in Figure 1). Time shifts were varied from 0 to 32 points, generating a symmetrical $32 \times 32$ matrix with the covariances as elements. (The first row was the autocovariation function itself, in the second row, elements were shifted by one column to the right, in the third row by two columns etc., so that the diagonal element was always the covariance with time lag zero.) The singular value decomposition (step 2 in Figure 1) generates 32 components that can be considered as dimensions of the state space; they include the signal and the noise. We assume that the variance due to the noise is lower than that due to the EEG signal. In order to separate noise as well as activity with little variance from the signal a cutoff criteria was chosen so that only eigenvectors were selected with eigenvalues larger than twice the smallest of all 32 eigenvalues. (This criterion has two advantages: it is independent of the particular gains used and it is easily reproducible among different laboratories. An alternative would be to choose a defined fraction of the largest eigenvalue or a criterion related to the total variance. The largest eigenvalue and consequently the total variance, however, may be sometimes completely dominated by very slow frequency waves. Then, such criteria would cut off faster than regular waves.) A subset of the eigenvectors was used to reconstruct the state space, the extracted eigenvectors defining the dimensionality of the space state (steps 3 and 4 in Figure 1).

In the next step the subset of the space state occupied by the attractor was determined using the method of "pointwise dimension" as proposed by Farmer et al (1983). Each point in the time series of the EEG can be placed in the multidimensional space (step 4 in Figure 1). Starting with a distinct reference point of the EEG time series, the number of points $N(\mathrm{r})$, which lie in a hypercube with radius $\mathrm{r}$ around this chosen point is counted. This counting is performed for subsequently larger radii until ultimately all points of the time series lie within this hypercube. (For a single-point attractor, the number of data points around the reference point (hypercube with radius $r$ ) will be 0 ; if the attractor is a one-dimensional line, the number of points will increase with $r^{1}$, for a two-dimensional 

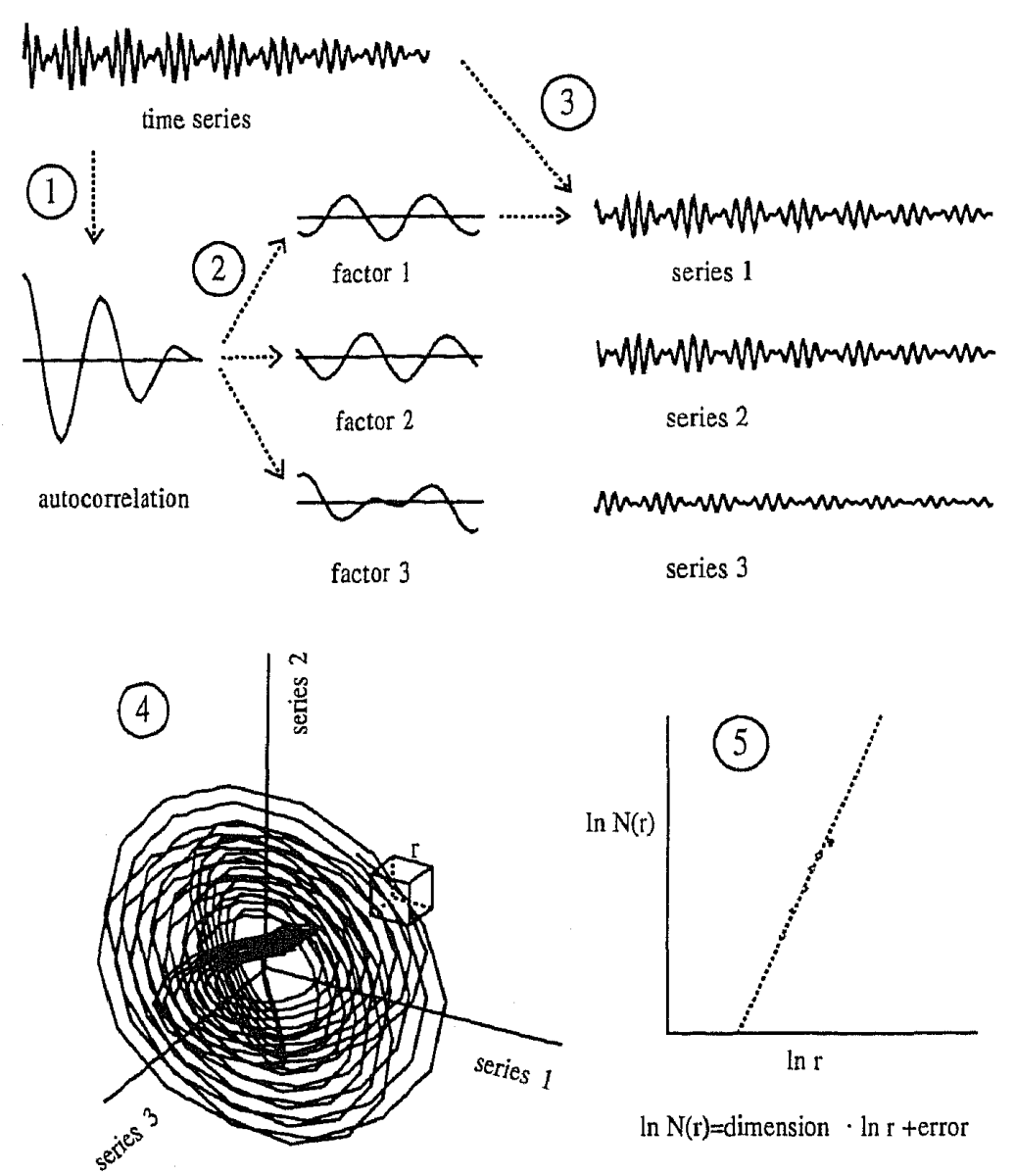

Figure 1. Schematic description of the procedure to estimate the dimensional complexity: The autocorrelation function is computed from the time series (1) and then decomposed into its principal components (2). For each point a linear sum of these factors is fitted to the time series (3). The corresponding weights determine a point in the state space (4). Moving along the time series, a trajectory is created. The dimension of this assembly of trajectories is estimated as dimensional complexity [also called "fractal dimension," (5)] A detailed description is provided in the text.

attraction, a plane, the number will increase with $r^{2}$, etc. Generally, the number of counted points will increase with $\mathrm{r}^{\mathrm{d}}$, with " $\mathrm{d}$ " denoting the "fractal dimension" of the attractor or "fractal complexity" of the EEG. ${ }^{2}$ For the present computations, 20 different radii $r_{i}$ were chosen. ${ }^{3}$ The distance between subsequent radii was selected such that each enlargement of the radius increased the total count by an equal number of points, that is, $N\left(\mathrm{r}_{\mathrm{i}}\right)-N\left(\mathrm{r}_{\mathrm{i}-1}\right)$ $=2048 / 20$. The counts are plotted against $\mathrm{r}$ using a double logarithmic scale. Then the points will lie on a straight line with the slope determining the "fractal dimension" $d$. Actually, the resulting function resembles a straight line of a certain slope only for intermediate radii, but declines parallel to the abscissa when the radii extend beyond the space covered by the attractor. A linear fit is performed on the straight segment, the slope of which is used for further calculation of the fractal dimension. (In order to obtain an estimation of the straight segment, the lowest ten values are chosen first. If the highest of these ten values has the largest distance to the straight line, the linear fit is recalculated

2"D" does not need to be an integer. In such cases we will refer to the attractor as "strange." Chaotic processes are characterized by such strange attractors.

${ }^{3}$ Usually, the radii are chosen to create equidistant differences on a logarithmic scale. This results in an S-like shape for the double logarithmic representation. The present method has the advantage that the lower curvature does not show up. 
for nine values only. If again the highest of these has the largest distance from the straight line, it is omitted from the next calculation. The process is repeated until the highest point no longer has the largest distance from the estimated straight line.). Typically, this procedure results in an estimate obtained from the five to seven lowest radii for the slope. After a slope has been determined for each of the reference points, the median of the slope values is taken as the measure of dimensional complexity.

These measures were averaged across the four selected EEG traces and then submitted to an analysis of variance with the between-subjects factor group (schizophrenic patients vs. control subjects) and the within-subject factors electrode ( $\mathrm{Fz}$ vs. $\mathrm{Cz}$ vs. $\mathrm{Pz}$ ) and condition (eyes open vs. eyes closed). $P$-values were obtained after adjustment of the degrees of freedom with the Greenhouse-Geisser $\varepsilon$. Means \pm SEM are presented. Associations with medication or clinical symptomatology were evaluated by regressional analyses.

\section{Results}

1. Parameters of the EEG-power spectra: For EEG-alpha the well-known topographical distribution (posterior predominance: electrode $F(2,44)=9.6, p<0.01$ ) and effect of the eyes-closed condition $[F(1,22)=12.4, p<0.01]$ were clearly visible and did not differ between groups.

2. Analysis of the EEG activity in the slow frequency band $(1-7 \mathrm{~Hz})$ uncovered a main effect electrode (with the highest amplitudes over the vertex: $F(2,44)=10.3$, $p<0.01$ ) and a main effect groups (with more pronounced activity in schizophrenics than in controls: $F(1,22)=15.1, p<0.01)$. Slow EEG activity was not related to either medication or symptomatology scores (affective flattening and cognitive impairment).

3. Mobility was enhanced under eyes-closed conditions compared to eyes open $[F(1,22)$ $=5.0, p<0.05]$. In controls this frequency was highest at the Pz electrode (particularly under the eyes-closed condition) which can be related to the appearance of alpha waves. The enhancement of alpha waves is also reflected in a significant main effect of electrode $[F(2,44)=11.2, p<0.01]$. Reduced frequency of alpha waves in patients was not sufficiently pronounced to produce a significant interaction of electrode $\times$ groups $[F(2,44)$ $=2.8, p<0.1]$. In patients the frequency of alpha waves in the frontal EEG were more pronounced with increasing scores of affective flattening $(r=0.59, p<0.05]$.

4. Hjorth complexity was smaller under "eyes closed" than under "eyes open" conditions $[F(1,22)=7.1, p<0.05]$. This was to be expected as more alpha waves will enhance the sinusoidality of the EEG waves. A trend for the interaction of electrode $\times$ group $[F(2,44)=2.7, p=0.1]$ with a significant difference between groups for the fronto-central gradient (parietal electrode omitted, $F(1,22)=11.6, p<0.01$ ) indicates that the sinusoidality of EEG waves recorded at the frontal and at the central site was about equal in controls, whereas in schizophrenic subjects, resemblance to sine waves was higher in the frontal EEG than over central areas (see Figure 2 top).

5. The dimensional complexity shows a very pronounced difference between groups in the topographical pattern: this measure generally gives larger values at the frontal than at the central sites in schizophrenic patients, whereas the reverse fronto-central gradient is exhibited by control subjects (group $\times$ electrode, $F(2,44)=4.2, p<0.05$; this interaction resides primarily in the fronto-central difference, as indicated by the analysis of variance (ANOVA) of the respective subset of the data $F(1,22)=14.5, p<0.01$ Fig. 2, bottom). As becomes evident from Figure 3 (bottom) eight of the twelve patients were beyond the range of controls' values (dotted area) for this fronto-central difference 

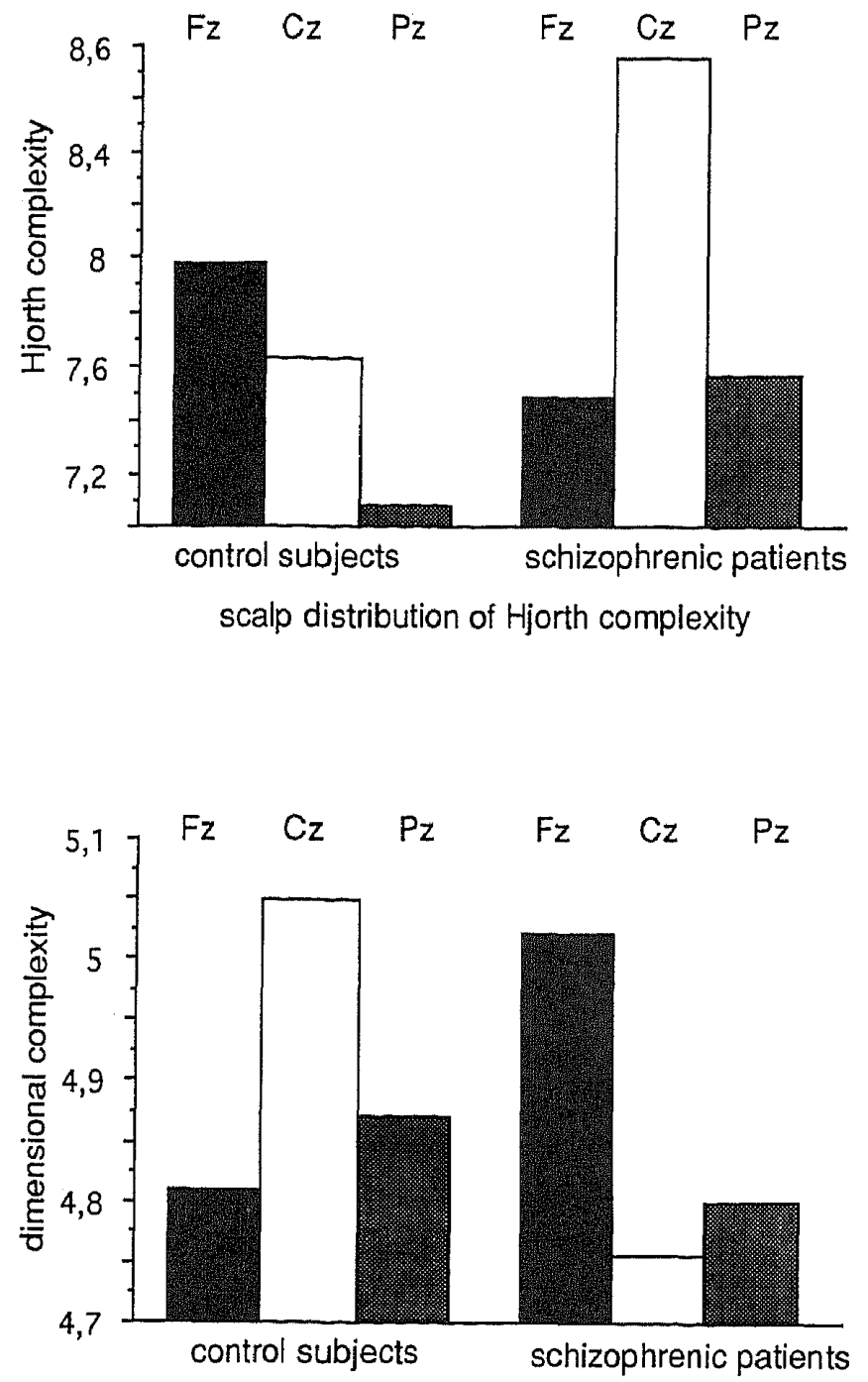

scalp distribution of dimensional complexity
Figure 2. Scalp distribution of the Hjorth complexity (top) and the dimensional complexity (bottom) in 12 control subjects (left group of bars) and 12 schizophrenic patients (right). The value for each measure is given on the ordinate. The acrosssubject averages for the frontal $(\mathrm{Fz})$, central $(\mathrm{Cz})$, and parietal $(\mathrm{Pz})$ recordings are plotted on the abscissa separately for the two groups.

(these eight patients are also outside the mean $\pm 2 \mathrm{SD}$; the mean is $-0.24 \pm 0.20$ ). The difference did not correlate with age, or-in the patient group-with medication, affective flattening, or cognitive impairment.

\section{Discussion}

In the present study, analysis of the EEG power spectra confirmed the common finding of increased activity in the slow-frequency range in schizophrenic patients (Zahn 1986; Itil 1976). Furthermore, examination of the dynamic features of the spontaneous EEG provided information additional to or even not available from traditional measures of EEG analyses. In particular, the scalp distribution or fronto-central gradient for Hjorth complexity and dimensional complexity were different in schizophrenic patients compared to patterns found in control subjects. Most schizophrenic patients exhibited higher frontal than central dimensional complexity values with two-thirds of the sample having values outside the range of the control group, half of the patients more than 3 SD above the 


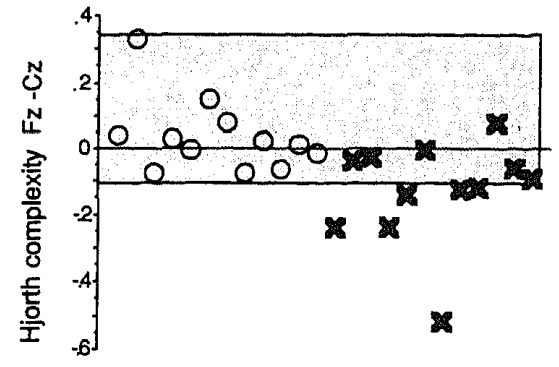

Ocontrol subjects $\mathbf{x}$ schizophrenic patients

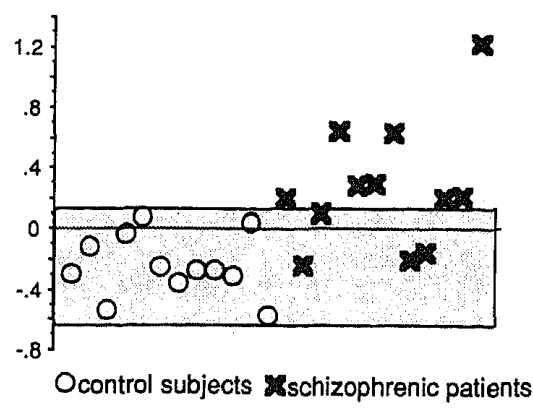

Figure 3. Distribution of the fronto-central gradient (difference between the frontal and central recording) for Hjorth complexity (top) dimensional complexity (bottom) for patients (crosses) and controls (open circles). Each symbol represents one subject. Dotted areas indicate the distribution range of control subjects to illustrate group differences.

mean of controls. (It is known that dimensionality is systematically underestimated. Therefore, the relationship of dimensional complexity between groups or conditions or recording sites is crucial rather than the particular value; Smith 1988).

If we assume that during rest the different brain regions are governed by the same dynamic processes, we may conclude that the dynamics of processing in the brain regions projecting to the frontal and central recording sites are different for most of the patients. It is well-known that the frontal recording not only picks up electrical activity generated in the frontal lobe, but also in the temporal lobes (e.g., supratemporal plane), which is volume-conducted to the $\mathrm{Fz}$ electrode. Therefore, we cannot conclude from the present measurements whether and to what extent the pattern of complexity in the patient group could be related to frontal and/or temporal deviances. In any case, the higher dimensional complexity values could be interpreted to indicate increased degrees of freedom of brain processes or a more complex dynamic in the respective areas in schizophrenic patients. Thus, even at rest, this group displays different attractors for brain electrical activity at one time. This pattern of fronto-central differences in dimensional complexity in schizophrenics as compared to controls seems to be of particular interest. Currently, we are aware of only three other group studies that reported topographical differences of phasespace attractors. Two investigations by Pritchard and colleagues (Pritchard et al 1991) and one from our group (Lutzenberger et al 1992) confirm fractal dimension of the EEGattractor, if at all different, to be lower at frontal than over central sites. Thus, the results for the control group are in line with previous findings. Data that we have collected from two alcoholics during withdrawal also demonstrated patterns in this normal range. Pritchard and coworkers did not report an unusual fronto-central gradient for elderly or demented (probably Alzheimer's) patients. The data base so far is certainly too limited 
to conclude that the finding of a higher frontal than central dimensional complexity indicates a specific characteristic in schizophrenic patients. The failure to uncover distinct patterns in alcoholic and demented patients may point to such a specificity, which, however, awaits further validation. If we compare this result with the repeatedly reported increased activity of slow EEG frequencies in schizophrenics, the fact that additional information is provided by the dimensional complexity measure becomes obvious. Although both results are statistically significant, the increase in the slow-frequency band has no topographical specificity and the overlap in the range of single-subject values between the patients and the control subjects is considerable.

A fronto-central difference was also depicted by the Hjorth complexity measure, suggesting more regularity in the appearance of brain waves over frontal areas in patients than in controls. Although it is possible to detect such a difference by visually examining the EEG traces, one would not easily predict that the higher regularity in the physical appearance of EEG-traces could indicate a more complex dynamic as expressed by the measure based on the attractor property. This fronto-centrally positive gradient of the fractal dimensionality of the EEG-attractor in schizophrenics indicates more intricacy of the brain dynamics (but not necessarily of the brain waves) over frontal than central areas.

None of the dynamic measures correlated with the neuroleptic dosage. (However, Itil 1977 also reported no effect of medication on alpha activity in patients.) Although the absence of correlation between dosage of medication and measures of EEG activity cannot be taken as a proof of the absence of drug effects, it is tempting to speculate that the presently observed patterns of complexity measures in patients cannot easily be attributed to effects of medication but adhere to the psychotic processes. However, this speculation must be substantiated by future comparisons of, example given, medicated and unmedicated patients. To our surprise, the distinctive EEG patterns in schizophrenic patients were also not related to the measures of symptomatology, affective flattening and cognitive impairment. It may, however, well be that these measures do not map abnormalities in the frontal and/or temporal brain dynamics in a comparable manner.

Although the present results are certainly preliminary, in particular because a new methodology was applied to EEG analysis in schizophrenics, their clarity encourage further investigation of the complexity of electrocortical responses in schizophrenics.

Two views of the cerebral cortex have a longstanding tradition. In one view the structural diversity of different areas and the bulk of connections being short in range divides the cortex into various subsystems. In an alternative view, statistical similarities, like the density of synapses, dendrites, and the existence of long-range connectivity and plasticity can be considered evidence for the system operating as a whole (Braitenberg 1986). As so often the solution to this problem seems to be dialectic in nature: there is some truth in both views, and like an electron, appearing one time as a wave, another time behaving like a particle, we may observe the cortex functioning as a whole system at one time, or separating into subsystems, according to different experimental conditions. This hypothesis receives support from the data reported by Lutzenberger et al (1992): although resting conditions or imagery of pleasant scenes yielded the same dimensional complexities at every electrode site, specific sensory tasks produced distinct patterns with different attractor dimensions across the scalp. When such differences are observed, we have reason to assume that the underlying dynamics must differ, thus arising from different systems at play at a time. The presently observed fronto-central gradients in patients classified as suffering from schizophrenia suggest that the frontal and the central dynamics 
are dissociated even in the relaxed waking state when a coupling occurs in the control sample.

Research was supported by the Deutsche Forschungsgemeinschaft. We gratefully acknowledge the assistance of R. Schlenker, P. Werther, and B. Vernon in assembling the psychometric data and physiological recordings.

\section{References}

Andreasen NG (1981): Scale for the Assessment of Negative Symptoms (SANS). Iowa City, University of Iowa.

Babloyantz A, Salazar JM, Nicolis C (1985)L Evidence of chaotic dynamics of brain activity during the sleep cycle. Physics Lett 11A:152-156.

Berg P (1986): The residual after correcting event-related potentials for blink artifacts. Psychophysiology 23:354-364.

Braitenberg V (1986): Two views of the cerebral cortex. In Palm G, Aertsen A (eds), Brain Theory. Berlin, Heidelberg, New York: Springer, pp 81-96.

Dvorak I, Siska J, Wackermann J, Hrudova L, Dostalek C (1986): Evidence for the interpretation of the EEG as a deterministic chaotic process with a low dimension. Activitas Nervosa Superior 28:228-231.

Farmer JD, Ott E, York JA (1983): Dimension of chaotic attractos. Physica 7D: 153-180.

Flor-Henry P (1988): EEG spectral analysis in psychopathology. In Giannitrapani D, Murri L (eds), The EEG of Mental Activities. Basel: Karger, pp 182-200.

Graf KE, Elbert T (1989): Dimensional analysis of the waking EEG. In Basar E, Bullock F (eds), Dynamics of Sensory and Cognitive Processing of the Brain. Berlin: Springer, pp 174-191.

Hjorth B (1975): Time domain descriptors and their relation to a particular model for generation of EEG activity. In Dolce G, Känkel $\mathrm{H}$ (eds), CEAN Computerized EEG Analyses. Stuttgart: Fischer, pp 3-8.

Hjorth B (1986): Physical aspects of EEG data as a basic for topographic mapping. In Duffy F (ed), Topographic Mapping of Brain Electrical Activity. Boston: Butterworth, pp 175-194.

Itil TM (1977): Qualitative and quantitative EEG findings in schizophrenia. Schizophr Bull 3:6175.

John R (1973): Where is fancy bred? In Hammer M, Salzinger K, Sutton S (eds), Psychopathology. New York: Wiley, pp 321-341.

Kay SR, Fiszbein A, Opler LA (1987): The positive and negative syndrome scale (PANSS) for schizophrenia. Schizophr Bull 13:261.

Koukkou M, Manske W (1986): Functional states of the brain and schizophrenic states of behavior. In Shagass C, Josiassen RC, Roemer RA (eds), Brain Electrical Potentials and Psychopathology. Amsterdam: Elsevier, pp 91-114.

Koukkou M, Tremel E, Manske M (1991): A psychobiological model of the pathogenesis of schizophrenic symptoms. Int J Psychophysiolo 10:203-212.

Layne SP, Mayer-Kressm G, Holzfuss J (1986): Problems associated with dimensional analysis of electroencephalogram data. In Mayer-Kress G (ed), Dimensions and Entropies in Chaotic Systems. New York: Springer, pp 246-256.

Lukoff D, Liberman RP, Nuechterlein KH (1986): Symptom monitoring in the rehabilitation of schizophrenic patients. Schizophr Bull 12:578-606.

Lutzenberger W, Elbert T, Birbaumer N, Ray WJ, Schupp H (1992): The scalp distribution of the fractal dimension of the EEG and its variation with mental tasks. Brain Topogr, 4 (in press).

Maurer K, Diercks T (1987): Brain mapping-topographische Darstellung des EEG und der evozierten Potentiale in Psychiatrie und Neurologie. Z EEG-EMG 18:4. 
Michelogiannis S, Paritsis N, Trikas P (1991): EEG coherence during hemispheric activation in schizophrenics. Eur Arch Pscyh Clin Neurosci 241:31-34.

Nan X, Jinghua $X$ (1988): The fractal dimension of EEG as a physical measure of conscious human brain activation. Bull Math Biol 50:559-565.

Pijn JP, Van Neerven J, Noestr A, Lopes da Silva FH (1991): Chaos or noise in the EEG signals: Dependence on state and brain site. Electroencephalogr Clin Neurophysiol 79:371-381.

Pritchard WS, Duke DW (1992a): Measuring chaos in the brain: A tutorial review of nonlinear dynamical EEG analysis. Int $J$ Neurosci (in press).

Pritchard WS, Duke DW (1992b): Measuring chaos in the brain: A tutorial review of EEG dimension estimation. Brain Cogn (in press).

Pritchard WS, Duke DW, Coburn KL (1991): Altered EEG dynamical responsivity associated with normal aging and probable Alzheimer's disease. Dementia 2:102-105.

Rapp PE, Zimmerman ID, Albano AM, de Guzman GC, Greenbaum NM, Bashore TE (1985): Experimental studies of chaotic neural behavior: Cellular activity and electroencephalographic signals. In Othmer G (ed), Nonlinear Oscillations in Chemistry and Biology. New York: Springer, pp 175-197.

Rapp PE, Bashore TE, Martinerie JM, Albano AM, Zimmerman ID, Mees A (1989): Dynamics of brain electrical activity. Brain Topogr 2:99-118.

Ray WR, Wells R, Elbert T, Lutzenberger W, Birbaumer N (1991): EEG and chaos: Dimensional estimation of sensory and hypnotic processes. In Duke D, Pritchard W (eds), Measuring Chaos in the Human Brain. World Scientific (in press).

Shagass C (1976): An electrophysiological view of schizophrenia. Biol Psychiatry 11:3-30.

Shagass C, Roemer RA, Straumanis JJ (1982): Relationships between psychiatric diagnosis and some quantitative EEG variables. Arch Gen Psychiatry 39:1423-1435.

Shagass C, Roemer RA, Straumanis JJ, Josiassen RD (1984): Psychiatric diagnostic discriminations with combinations of quantitative EEG variables. Br J Psychiatry 144:581-592.

Smith LA (1988) Physics Lett A133:283.

Watt RC, Hameroff SR (1987): Phase space analysis of human EEG during general anesthesia. In Koslow SH (ed), Perspectives in Biological Dynamics and Theoretical Medicine. New York: Academy of Sciences, pp 286-288.

World Health Organization (1980): Psychological Impairment Rating Schedule: International Classification of Impairments, Disabilities and Handicaps. Geneva: WHO.

Zahn TP (1986): Psychophysiological approaches to psychopathology. In Coles MGH, Donchin E, Porges S (eds), Handbook of Psychophysiology. New York: Plenum Press, pp 508-610. 